\title{
Heat shock protein 90 in neurodegenerative diseases
}

\author{
Wenjie Luo', Weilin Sun², Tony Taldone2, Anna Rodina² and Gabriela Chiosis*2
}

\begin{abstract}
Hsp90 is a molecular chaperone with important roles in regulating pathogenic transformation. In addition to its wellcharacterized functions in malignancy, recent evidence from several laboratories suggests a role for Hsp90 in maintaining the functional stability of neuronal proteins of aberrant capacity, whether mutated or over-activated, allowing and sustaining the accumulation of toxic aggregates. In addition, Hsp90 regulates the activity of the transcription factor heat shock factor-1 (HSF-1), the master regulator of the heat shock response, mechanism that cells use for protection when exposed to conditions of stress. These biological functions therefore propose Hsp90 inhibition as a dual therapeutic modality in neurodegenerative diseases. First, by suppressing aberrant neuronal activity, Hsp90 inhibitors may ameliorate protein aggregation and its associated toxicity. Second, by activation of HSF-1 and the subsequent induction of heat shock proteins, such as Hsp70, Hsp90 inhibitors may redirect neuronal aggregate formation, and protect against protein toxicity. This mini-review will summarize our current knowledge on Hsp90 in neurodegeneration and will focus on the potential beneficial application of Hsp90 inhibitors in neurodegenerative diseases.
\end{abstract}

\section{Roles of Hsp90 in neurodegeneration}

Hsp90 is a molecular chaperone with important roles in maintaining the functional stability and viability of cells under a transforming pressure [1-3]. For neurodegenerative disorders associated with protein aggregation, the rationale has been that inhibition of $\mathrm{Hsp} 90$ activates heat shock factor-1 (HSF-1) to induce production of Hsp70 and Hsp40, as well as of other chaperones, which in turn, promote disaggregation and protein degradation [4-6]. However, recent evidence reveals an additional role for Hsp90 in neurodegeneration. Namely, Hsp90 maintains the functional stability of neuronal proteins of aberrant capacity, thus, allowing and sustaining the accumulation of toxic aggregates $[7,8]$. Below, we summarize the current understanding on these Hsp90 biological roles and review potential applications of pharmacological Hsp90 inhibition in neurodegenerative diseases.

\section{HSF-1 is a master regulator of the heat shock response} Exposed to conditions of stress, cells normally respond by activation of the heat shock response (HSR) accompanied

* Correspondence: chiosisg@mskcc.org

2 Department of Medicine and Program in Molecular Pharmacology and Chemistry, Memorial Sloan-Kettering Cancer Center, New York, NY 10021, USA Full list of author information is available at the end of the article by increased synthesis of a number of cytoprotective heat shock proteins (Hsps) which dampen cytotoxicity, such as caused by misfolded and denatured proteins [4-6]. The most prominent part of this transition occurs on the transcriptional level. In mammals, protein-damaging stress is regulated by activation of HSF-1, which binds to upstream regulatory sequences in the promoters of heat shock genes [9]. The activation of HSF-1 proceeds through a multi-step pathway, involving a monomer-totrimer transition, nuclear accumulation and extensive posttranslational modifications (Fig. (1A)). The function of HSF-1 is regulated by Hsp90 [10]. Namely, under nonstressed conditions, Hsp90 binds to HSF-1 and maintains the transcription factor in a monomeric state. Stress, heat shock or inhibition of Hsp90 release HSF-1 from the Hsp90 complex, which results in its trimerization (Fig. (1B)), activation and translocation to the nucleus where it initiates a heat shock response, manifested in the production of Hsps such as the chaperones Hsp70 and its activator, Hsp40 (Fig. $(1 \mathrm{~A}, \mathrm{C})$ ). Neurons in the differentiated state, both in vivo and in vitro systems have been reported to be resistant to Hsp induction following conventional heat shock [5]. In contrast, pharmacologic induction of Hsp70 upon HSF-1 activation has been documented, and moreover as described below, demon- 


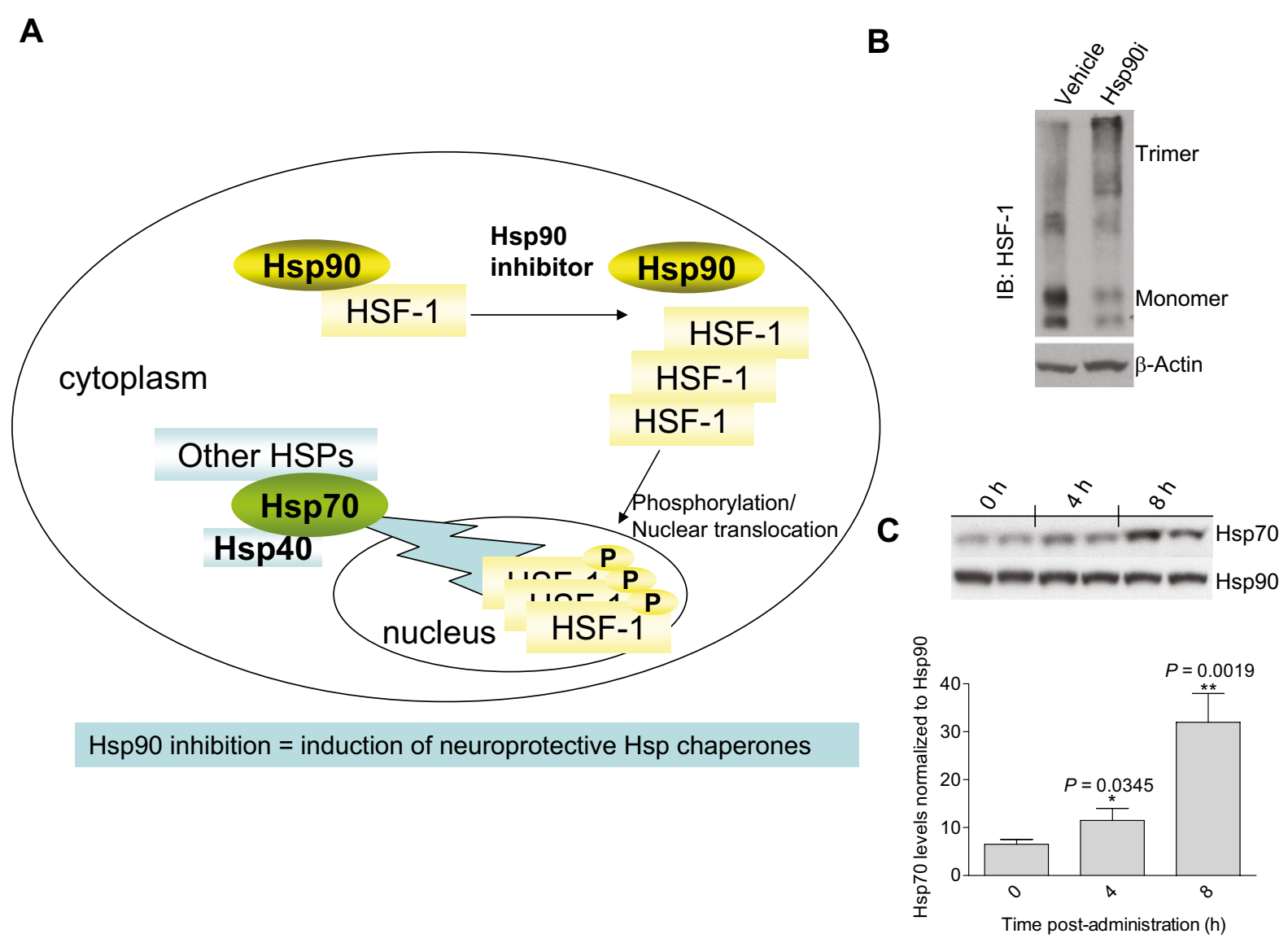

Figure 1 Heat shock proteins are induced upon Hsp90 inhibition. (A) Schematic representation of HSF-1 regulation by Hsp90 and its activation by Hsp90 inhibitors. (B) Treatment of cells with heat shock or an Hsp90 inhibitor (Hsp90i) results in HSF-1 trimerization [10]. (C) Systemic administration of the purine-scaffold Hsp90 inhibitor PU-DZ8 to AD transgenic mice results in Hsp70 induction in the brain [29].

strated to be protective in neurons against toxicity caused by multiple types of insults.

\subsection{Hsp70 redirects neuronal aggregate formation and protects against aggregate toxicity}

Frequently, neurodegenerative diseases are characterized by a gain of toxic function of misfolded proteins. Here, toxicity may result from an imbalance between normal chaperone capacity and production of dangerous protein species. Increased chaperone expression can suppress the neurotoxicity of these molecules, suggesting possible therapeutic strategies. Indeed, several studies summarized below, have reported a reduction in cellular toxicity upon expression of Hsp70 and Hsp40 in neurodegenerative aggregation disease models.

The polyglutamine (polyQ) diseases consist of nine neurodegenerative diseases in which a polyQ tract expansion leads to protein misfolding and subsequent deposition of protein aggregates in neurons [11]. Among these are Huntington's disease (HD), spinal and bulbar muscular atrophy (SBMA), Dentatorubral-pallidoluysian atrophy (DRPLA), and several ataxias (SCA1-3). In HD, mutant forms of huntingtin (htt) with expanded glutamine repeats form nuclear and cytoplasmic aggregates. Muchowski et al found that Hsp70 and its cochaperone Hsp40 suppressed the assembly of htt into spherical and annular polyglutamine oligomers and thus attenuated the formation of detergent-insoluble amyloid-like fibrils [12]. Likewise, in a yeast model of HD, expression of Hsp70 and Hsp40 reduced the toxicity associated with expression of mutant htt by preventing its aberrant interaction with an essential polyQ-containing transcription factor [13]. Studies in a mouse model of HD suggested that in neurons, protection by Hsp70 against the toxic effects of misfolded htt protein occurred by mechanisms independent of the deposition of fibrillar aggregates, namely by binding monomeric and/or low molecular mass SDS-sol- 
uble oligomers that are likely off-pathway to fibril formation, but may be potentially pathogenic [14]. In a mammalian model of spinocerebellar ataxia (SCA) type 1, expression of Hsp70 afforded protection against polyQinduced neurodegeneration [15]. Pharmacological induction of heat-shock proteins in spinal and bulbar muscular atrophy (SBMA)-transgenic mice suppressed nuclear accumulation of the pathogenic androgen receptor (AR) protein, resulting in amelioration of polyglutaminedependent neuromuscular phenotypes [16].

In amyotrophic lateral sclerosis (ALS), the FDAapproved drug riluzole was reported to partly act by HSF1 activation and amplification of the HSR [17]. In the Superoxide dismutase 1 (SOD1) mouse model of ALS, elevation in levels of Hsp70 by another agent, arimoclomol, protected motor-neurons in both acute injuryinduced motor-neuron degeneration as well as progressive motor-neuron degeneration models [18].

In various cellular models of Alzheimer's disease (AD), increased levels of Hsp70 promoted tau solubility and tau binding to microtubules [19]. Hsp70 also inhibited the propensity of $A \beta$ to aggregate [20], and reduced the toxicity of $A \beta$ on neuronal cultures [21]. Moreover, Amyloid precursor protein (APP) and/or its amyloidogenic derivative $\mathrm{A} \beta$ are targets of chaperone mediated clearance [22,23]. In Drosophila melanogaster and yeast models of Parkinson's disease (PD), directed expression of Hsp70 or pharmacologic Hsp modulation prevented the neuronal loss caused by $\alpha$-synuclein $[24,25]$. Huang et al reported that these effects of Hsp70 manifested by inhibition of $\alpha$ synuclein fibril formation via preventing the formation of prefibrillar $\alpha$-synuclein formation [26]. Using $\alpha$-synuclein deletion mutants, Luk et al indicated that interactions between the Hsp70 substrate binding domain and the $\alpha$ synuclein core hydrophobic region mediated assembly inhibition, and that the assembly process was inhibited prior to the elongation stage [27].

Overall, in the several neurodegenerative disease models presented above, overexpression of Hsp70 improved the severities of several disease phenotypes without visibly affecting aggregate formation, suggesting that chaperones do not prevent aggregation per se, but rather redirect aggregates into amorphous deposits, thereby sequestering potentially toxic species from bulk solution.

\subsection{Expression of Hsp70 and other Hsps are induced upon pharmacologic Hsp90 inhibition}

As noted above, inhibition of Hsp90 releases HSF-1 from the Hsp90 complex resulting in subsequent production of Hsps (Fig. (1)), and induction of Hsp70 by Hsp90 inhibitors is well documented in neurodegenerative disease models. Geldanamycin (GM) (Fig. (2)), an Hsp90 inhibitor [28], induced a dose-dependent increase of Hsp70 in an AD cell model, as well as in rat primary cortical neurons [19] and reduced the amount of insoluble tau and

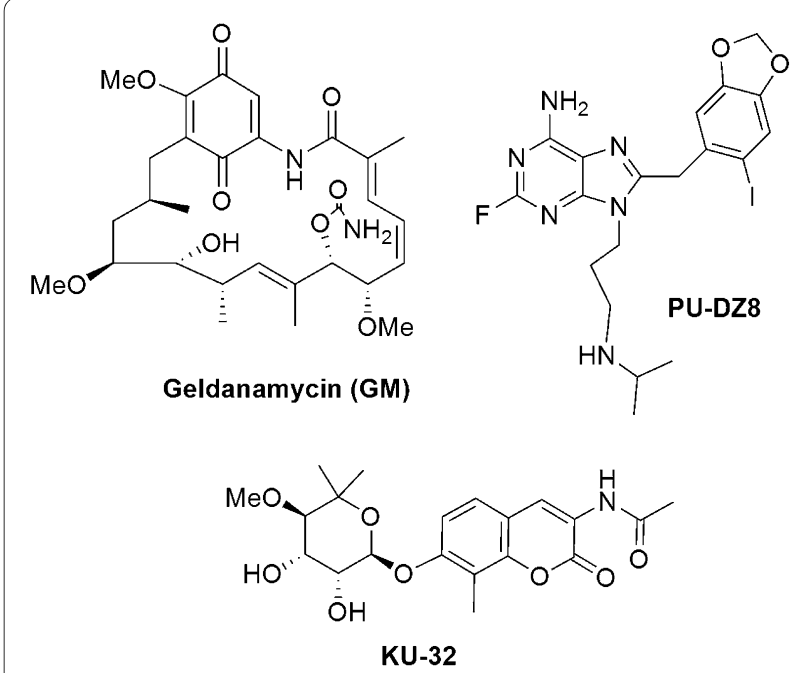

Figure 2 Chemical structures of several representative Hsp90 inhibitors. GM = ansamycin class; PU-DZ8 = purine-scaffold class; $\mathrm{KU}-32$ $=$ novobiocin class

the basal levels of okadaic-acid induced tau phosphorylation [19]. Treatment of primary cortical neurons with the purine-scaffold Hsp90 inhibitor PU24FCl, led to a dosedependent increase in Hsp70 [29]. Similarly, administration of two CNS-permeable PU24FCl-derivatives, PUDZ8 (Fig. (2)) [29] and EC102 [30], to tau transgenic mice (htau and JNPL3) resulted in Hsp70 induction in the brain, effects maintained at $24 \mathrm{~h}$ post-administration. KU32, an Hsp90 inhibitor of distinct chemical nature (Fig. (2)), induced Hsp70 in SH-SY5Y neuroblastoma cultures and protected them against $A \beta$-induced toxicity [31]. GM activated a heat shock response and inhibited htt aggregation in a cell culture model of HD [32,33] and induced Hsp70 in a time- and concentration-dependent manner and prevented $\alpha$-synuclein aggregation and protected against toxicity in a cellular $\alpha$-synuclein aggregation model [34]. Auluck et al reported that treatment of a fly model of PD with GM fully protected against $\alpha$-synuclein toxicity [35]. GM also protected against 1-methyl-4pheny-1,2,3,6-tetrahydropyridine (MPTP)-induced dopaminergic neurotoxicity, a mouse model of PD [36]. Pretreatment with GM via intracerebral ventricular injection, prior to MPTP treatment, induced Hsp70 and increased residual dopamine content and tyrosine hydroxylase immunoreactivity in the striatum [36]. Hsp70 induction in the spinal cord was noted upon intraperitoneal injection of a GM derivative, 17-AAG, in a mouse model of SBMA [37]. 17-AAG was also effective against neurodegeneration in other polyQ diseases [38]. Namely, it suppressed compound eye degeneration and inclusion body formation and rescued the lethality in a Drosophila model of SCA. It also suppressed neurodegeneration in a HD fly model. Knockdown of HSF-1 abol- 
ished the induction of molecular chaperones and the therapeutic effect of 17-AAG on polyQ-induced neurodegeneration in the Drosophila models, arguing that the therapeutic effect of 17-AAG was mainly HSF1-mediated [38].

In summary, HSF-1 activation by Hsp90 inhibitors was noted in several in vitro and in vivo models of neurodegenerative disease, suggesting Hsp90 inhibition as a means to modulate Hsp levels in the diseased brain, with the goal of protecting against the toxic proteins that arise during the neurodegenerative process.

\section{Inhibition of Hsp90 reduces aberrant neuronal protein activity and expression}

In addition to regulation of HSF-1, recent evidence suggests an additional role for Hsp90 in maintaining the functional stability of neuronal proteins of aberrant capacity (Fig. (3A)).

SBMA is an inherited motor neuron disease caused by the expansion of a polyglutamine tract within the androgen receptor (AR) [11]. The pathologic features of SBMA are motor neuron loss in the spinal cord and brainstem and diffuse nuclear accumulation and nuclear inclusions of the mutant AR (mAR) in the residual motor neurons and certain visceral organs. Waza et al demonstrated that $\mathrm{mAR}$ present in SBMA is one of the proteins regulated by Hsp90 (Fig. (3A)) [37]. Hsp90 formed a molecular complex with mAR to maintain its functional stability. In both SBMA cell models and transgenic mice, inhibition of Hsp90 by 17-AAG led to a preferential degradation of the mAR, mainly by the proteasome machinery. These effects of 17-AAG were uncoupled from induction of Hsp70, and resulted from direct destabilization of $\mathrm{mAR}$ and its subsequent degradation upon Hsp90 inhibition. In a transgenic mouse model of SBMA, 17-AAG ameliorated motor impairments without detectable toxicity, and reduced the amounts of monomeric and aggregated mAR [37]. Similar findings were reported by Thomas et al; these authors found that pharmacologic Hsp90 inhibition blocked the development of aggregates of the expanded glutamine androgen receptor (AR112Q) in HSF1(-/-) mouse embryonic fibroblasts where Hsp70 and Hsp40 chaperones were not induced [39].

Parkinson disease (PD), the most common neurodegenerative movement disorder, is characterized by a complexity of pathogenic events [40], many of which were recently linked to Hsp90 (Fig. (3A)). Wang et al have recently shown that Leucine-rich repeat kinase 2 (LRRK2), a kinase of whose mutated forms is prevalent in both familial and apparently sporadic cases of PD, formed a complex with Hsp90 in vivo [41]. Inhibition of Hsp90 function by the purine-scaffold Hsp90 inhibitor PU-H71 disrupted the association of Hsp90 with LRRK2 and led to elimination of LRRK2 by the proteasome. PU-H71 lim- ited the mutant LRRK2-elicited toxicity to neurons and rescued the axon growth retardation defect caused by the LRRK2 G2019S mutation in neurons [41]. Mutation of PTEN-induced kinase 1 (PINK1), which encodes a putative mitochondrial serine/threonine kinase, leads to PARK6, an autosomal recessive form of familial Parkinson's disease [40]. The recessive inheritance of this form of Parkinson's disease suggests loss of PINK1 function is closely associated with its pathogenesis. Moriwaki et al have reported that Hsp90 binds PINK1 to enhance its stability. In cells treated with the Hsp90 inhibitor GM, levels of PINK1 were greatly diminished via the ubiquitin-proteasome pathway [42]. $\alpha$-Synuclein is an intrinsically unstructured protein that may form fibrils, and is also involved in PD neurodegeneration [40]. Falsone et al has recently reported that Hsp90 influences $\alpha$-synuclein vesicle binding and amyloid fibril formation, two processes that are tightly coupled to $\alpha$-synuclein folding [43]. Namely, Hsp90 bound $\alpha$-synuclein and abolished the interaction of this polypeptide with small unilamellar vesicles. Hsp90 also promoted fibril formation in an ATPdependent manner via oligomeric intermediates [43]. Another link between $\alpha$-synuclein and Hsp90 was provided by Kabuta et al [44]. Alpha-synuclein is degraded at least partly by chaperone-mediated autophagy (CMA). The authors suggested that aberrant interaction of mutant ubiquitin C-terminal hydrolase L1 (UCH-L1) with the chaperone-mediated autophagy CMA machinery, at least partly accounted for the pathogenesis of PD associated with I93M UCH-L1 and the increase in the amount of $\alpha$-synuclein [44].

In tauopathies, neurodegenerative diseases characterized by tau protein abnormalities, transformation is characterized by abnormalities in the tau protein leading to an accumulation of hyperphosphorylated and aggregated tau [45]. In AD, tau hyperphosphorylation is suggested to be a pathogenic process caused by aberrant activation of several kinases, in particular cyclin-dependent protein kinase 5 (CDK5) and glycogen synthase kinase-3 beta (GSK3 $\beta)$, leading to phosphorylation of tau on pathogenic sites [46]. Hyperphosphorylated tau in $\mathrm{AD}$ is believed to misfold, undergo net dissociation from microtubules and form toxic tau aggregates. In a cluster of tauopathies termed "frontotemporal dementia and parkinsonism linked to chromosome 17 (FTDP-17)", transformation is caused by several mutations in human tau isoforms on chromosome 17, that result in and are characterized by the accumulation of aggregated tau similar to that in AD [47]. Luo et al have reported that the stability of p35 and p25, neuronal proteins that activate CDK5 through complex formation leading to aberrant tau phosphorylation, and that of mutant but not wild type tau protein, were maintained in tauopathies by Hsp90 (Fig. (3A)) [29]. Inhibition of Hsp90 in both cellular and mouse 
A

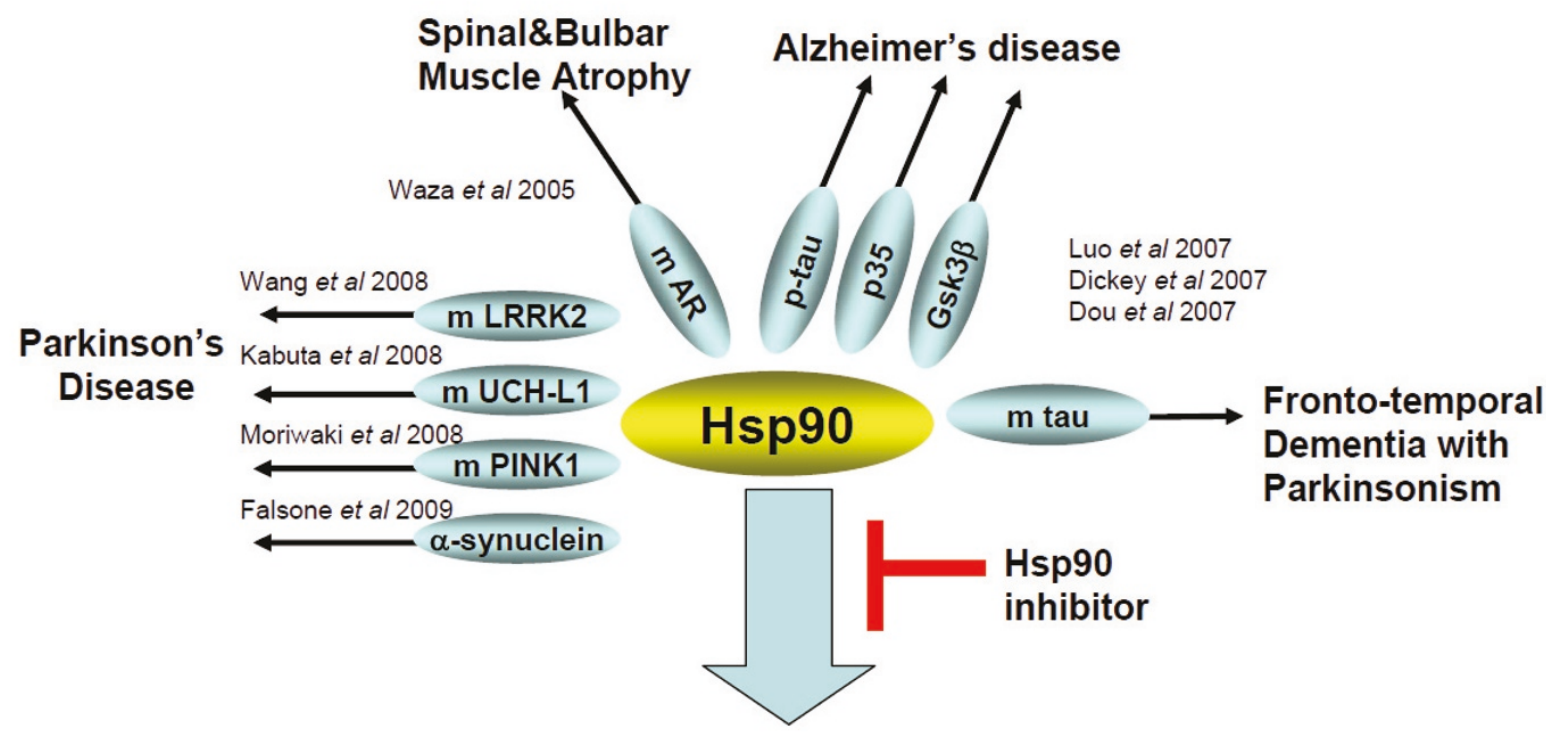

Hsp90 inhibition = reduction in aberrant neuronal protein activity and expression

B
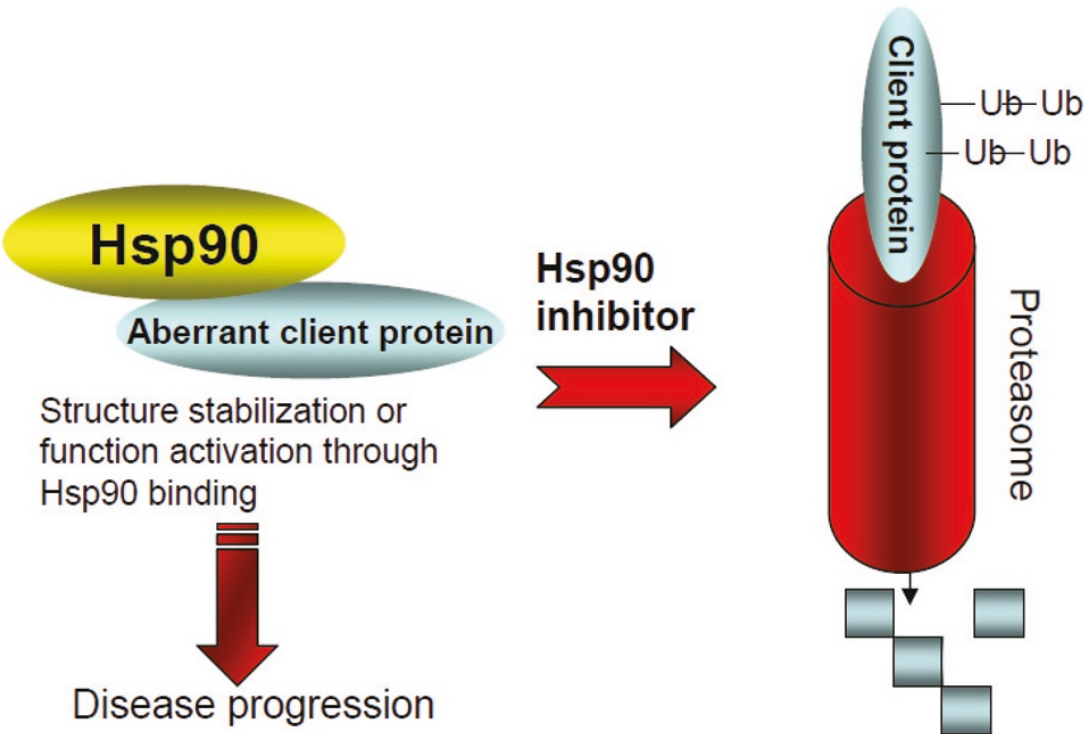

Figure $3 \mathrm{Hsp} 90$ shelters aberrant neuronal proteins. (A) Aberrant neuronal proteins regulated by Hsp90. To tolerate the accumulation of dysregulated processes and to allow the development of the disease phenotype, the functional stability of these aberrant processes likely requires a "buffering" mechanism, such as offered by Hsp90. These aberrant neuronal proteins activities develop Hsp90-dependency and promote disease progression. (B) Pharmacologic Hsp90 inhibition results in inactivation or degradation of Hsp90-regulated proteins, mainly by a proteasomal pathway. 
models of tauopathies led to reduction of the pathogenic activity of these proteins and resulted in a dose- and time-dependent elimination of aggregated tau [29]. When administered 5xweek for 30 days to JNPL3 transgenic (tg) mice, PU-DZ8 (Fig. (2), led to significant reduction in mutant tau expression and phosphorylation without toxicity to the mice [29]. Complementarily, Dickey et al demonstrated that the EC102 Hsp90 inhibitor promoted selective decrease in ptau species in a tg mouse model of AD independent of HSF-1 activation (Fig. (3A)) [30]. Both reports identify the proteasomal pathway as responsible for degradation of the aberrant tau species following Hsp90 inhibition [29,30]. A link between Hsp90 and GSK3 $\beta$ was reported by Dou et al (Fig. (3A)) [48]. Namely, the stability and function of the GSK3 $\beta$ was found to be maintained by Hsp90, and Hsp90 inhibition by GM and PU24FCl led to a reduction in the protein level of GSK3 $\beta$, effect associated with a decrease in tau phosphorylation at putative GSK3 $\beta$ sites [48]. Further, Tortosa et al reported that binding of Hsp90 to tau facilitates a conformational change in tau that could result in its phosphorylation by GSK3 and its aggregation into filamentous structures [49].

Collectively, the above data suggest that at the phenotypic level, Hsp90 appears to serve as a biochemical buffer for the numerous aberrant processes that facilitate the evolution of the neurodegenerative phenotype (Fig. (3A)). Inhibition of Hsp90 by small molecules results in the destabilization of the Hsp90/aberrant protein complexes leading primarily to degradation of these proteins by a proteasome-mediated pathway (Fig. (3B)).

\section{Conclusion}

Collectively, these reports suggest that in neurodegenerative diseases Hsp90 inhibition may offers a dual therapeutic approach. On one hand, its benefits may come from induction of Hsp70 and other chaperones able of redirecting neuronal aggregate formation, and capable of protective potential against protein toxicity, proposing Hsp90 inhibition as a pharmacological intervention to therapeutically increase expression of molecular chaperone proteins to treat neurodegenerative diseases where aggregation is central to the pathogenesis (Fig. (1)). On the other hand, Hsp90 inhibition may ameliorate protein hyperphosphorylation and subsequent aggregation by reduction of aberrant neuronal protein activity (Fig. (3)). The usefulness of Hsp90 inhibitors as clinical agents in neurodegenerative diseases will depend on whether their effects occur at concentrations of drug that are not toxic and on whether the drugs can be administered chronically in such a fashion so as to safely achieve these concentrations in the brain. While studies in several cellular models show promise for this class of compounds in treating a large spectrum of neurodegenerative diseases, these studies need to be furthered in animal models, with the goal of testing both Hsp90 inhibitors efficacy in improving neuro-pathology and their safety under longterm administration schedules. While several of the studies have used GM and its derivatives, these agents have several liabilities that limit their future clinical use [50]. Development for cancers of Hsp90 inhibitors of scaffolds distinct from that of GM is currently reaching an explosive phase, where several agents are in clinical evaluation, with many others following behind [50]. It is likely that the Hsp90 inhibitor classes with best safety profiles will also move into the neurodegenerative space. It now remains the goal of medicinal chemistry to deliver CNSpermeable Hsp90 inhibitors with a good therapeutic index to fulfill the promise of these agents in the treatment of neurodegenerative diseases.

\section{List of abbreviations}

AD: Alzheimer's disease; APP: amyloid precursor protein; ALS: amyotrophic lateral sclerosis; AR: androgen receptor; CDK5: cyclin-dependent protein kinase 5; CMA: chaperone-mediated autophagy; CNS: central nervous system; DRPLA: Dentatorubral-pallidoluysian atrophy; FTDP-17: frontotemporal dementia and parkinsonism linked to chromosome 17; FDA: Food and Drug Administration; GM: Geldanamycin; GSK3ß: glycogen synthase kinase-3 beta; HD: Huntington's disease; HSF-1: heat shock factor-1; HSR: heat shock response; Hsps: heat shock proteins; Hsp90: Heat shock protein 90; Hsp70: Heat shock protein 70; Hsp90i: Hsp90 inhibitor; Htt: huntingtin; LRRK2: Leucine-rich repeat kinase 2; MPTP: 1-methyl-4-pheny-1,2,3,6-tetrahydropyridine; PARK6: Parkinson disease 6: autosomal recessive earlyonset; PD: Parkinson disease; PolyQ: polyglutamine diseases; PINK1: PTEN-induced kinase 1; SBMA: spinal and bulbar muscular atrophy; SCA: spinocerebellar ataxia; SDS: sodium dodecyl sulfate; SOD1: Superoxide dismutase 1; Tg: transgenic; UCH-L1: ubiquitin C-terminal hydrolase L1;

\section{Competing interests \\ The authors are inventors on patents and patent applications relating to Hsp90 compositions of matter and to the use oh Hsp90 inhibitors in neurodegenera- tive diseases.}

\section{Authors' contributions}

All authors contributed to the concept and writing the review article, and read and approved the final manuscript.

\section{Acknowledgements}

This work was partially funded by Association for Frontotemporal Dementias and the Alzheimer's Drug Discovery Foundation, the Institute of Aging, Grant\#281207, and the National Institute of Aging, grants 5-R21AG028811-2 and 1 U01 AG032969-01A1, and NIA K01 AG032364-01A2. 


\section{Author Details}

'Laboratory of Molecular and Cellular Neuroscience, The Rockefeller University and Fisher Foundation for Alzheimer's Disease, New York, NY 10021, USA and 2Department of Medicine and Program in Molecular Pharmacology and Chemistry, Memorial Sloan-Kettering Cancer Center, New York, NY 10021, USA

Received: 23 March 2010 Accepted: 3 June 2010

Published: 3 June 2010

\section{References}

1. Whitesell L, Lindquist SL: HSP90 and the chaperoning of cancer. Nat Rev Cancer 2005, 5:761-772.

2. Workman P, Burrows F, Neckers L, Rosen N: Drugging the cancer chaperone HSP90: combinatorial therapeutic exploitation of oncogene addiction and tumor stress. Ann N Y Acad Sci 2007, 1113:202-216

3. Chiosis G: Targeting chaperones in transformed systems - a focus on Hsp90 and cancer. Expert Opin Ther Targets 2006, 10:37-50.

4. Klettner $\mathrm{A}$ : The induction of heat shock proteins as a potential strategy to treat neurodegenerative disorders. Drug News Perspect 2004, 17:299-306.

5. Brown I: Heat Shock Proteins and Protection of the Nervous System. Ann N Y Acad Sci 2007, 1113:147-158.

6. Muchowski PJ, Wacker JL: Modulation of neurodegeneration by molecular chaperones. Nat Rev Neurosci 2005, 6:11-22.

7. Waza M, Adachi H, Katsuno M, Minamiyama M, Tanaka F, Doyu M, Sobue G: Modulation of Hsp90 function in neurodegenerative disorders: a molecular-targeted therapy against disease-causing protein. $\mathrm{J} \mathrm{Mol}$ Med 2006, 84:635-646.

8. Luo W, Rodina A, Chiosis G: Heat shock protein 90: translation from cancer to Alzheimer's disease treatment? BMC Neurosci 2008, 9(Suppl 2):S7.

9. Anckar J, Sistonen L: Heat shock factor 1 as a coordinator of stress and developmental pathways. Adv Exp Med Biol 2007, 594:78-88.

10. Zou J, Guo Y, Guettouche T, Smith DF, Voellmy R: Repression of heat shock transcription factor HSF1 activation by HSP90 (HSP90 complex) that forms a stress-sensitive complex with HSF1. Cell 1998, 94:471-480.

11. Williams AJ, Paulson HL: Polyglutamine neurodegeneration: protein misfolding revisited. Trends Neurosci 2008, 31:521-528.

12. Muchowski PJ, Schaffar G, Sittler A, Wanker EE, Hayer-Hartl MK, Hartl FU: $\mathrm{Hsp} 70$ and hsp40 chaperones can inhibit self-assembly of polyglutamine proteins into amyloid-like fibrils. Proc Natl Acad Sci USA 2000, 97:7841-7846.

13. Krobitsch S, Lindquist S: Aggregation of huntingtin in yeast varies with the length of the polyglutamine expansion and the expression of chaperone proteins. Proc Natl Acad Sci USA 2000, 97:1589-1594.

14. Wacker JL, Huang SY, Steele AD, Aron R, Lotz GP, Nguyen Q, Giorgini F, Roberson ED, Lindquist S, Masliah E, Muchowski PJ: Loss of Hsp70 exacerbates pathogenesis but not levels of fibrillar aggregates in a mouse model of Huntington's disease. J Neurosci 2009, 29:9104-9114.

15. Cummings $C J$, Mancini MA, Antalffy B, DeFranco DB, Orr HT, Zoghbi HY: Chaperone suppression of aggregation and altered subcellular proteasome localization imply protein misfolding in SCA1. Nat Genet 1998, 19:148-154

16. Katsuno M, Sang C, Adachi H, Minamiyama M, Waza M, Tanaka F, Doyu M, Sobue G: Pharmacological induction of heat-shock proteins alleviates polyglutamine-mediated motor neuron disease. Proc Natl Acad Sci USA 2005, 102:1606-1680.

17. Yang J, Bridges $K$, Chen KY, Liu AY: Riluzole increases the amount of latent HSF1 for an amplified heat shock response and cytoprotection. PLoS One 2008, 3:e2864.

18. Kieran D, Kalmar B, Dick JR, Riddoch-Contreras J, Burnstock G, Greensmith $\mathrm{L}$ : Treatment with arimoclomol, a coinducer of heat shock proteins, delays disease progression in ALS mice. Nat Med 2004, 10:402-405.

19. Dou F, Netzer WJ, Tanemura K, Li F, Hartl FU, Takashima A, Gouras GK, Greengard P, Xu H: Chaperones increase association of tau protein with microtubules. Proc Natl Acad Sci USA 2003, 100:721-726.

20. Evans CG, Wisen S, Gestwicki JE: Heat shock proteins 70 and 90 inhibit early stages of amyloid $\beta$-(1-42) aggregation in vitro. J Biol Chem 2006, 281:33182-33191.

21. Ansar S, Burlison JA, Hadden MK, Yu XM, Desino KE, Bean J, Neckers L, Audus KL, Michaelis ML, Blagg BS: A non-toxic Hsp90 inhibitor protects neurons from A $\beta$-induced toxicity. Bioorg Med Chem Lett 2007, 17:1984-1990

22. Kumar P, Ambasta RK, Veereshwarayya V, Rosen KM, Kosik KS, Band H, Mestril R, Patterson C, Querfurth HW: CHIP and HSPs interact with betaAPP in a proteasome-dependent manner and influence Abeta metabolism. Hum Mol Genet 2007, 16:848-864

23. Kouchi Z, Sorimachi H, Suzuki K, Ishiura S: Proteasome inhibitors induce the association of Alzheimer's amyloid precursor protein with $\mathrm{Hsc73}$. Biochem Biophys Res Commun 1999, 254:804-810.

24. Auluck PK, Chan HY, Trojanowski JQ, Lee VM, Bonini NM: Chaperone suppression of alpha-synuclein toxicity in a Drosophila model for Parkinson's disease. Science 2002, 295:865-868.

25. Flower TR, Chesnokova LS, Froelich CA, Dixon C, Witt SN: Heat shock prevents alpha-synuclein-induced apoptosis in a yeast model of Parkinson's disease. J Mol Biol 2005, 351:1081-1100.

26. Huang C, Cheng H, Hao S, Zhou H, Zhang X, Gao J, Sun QH, Hu H, Wang CC: Heat shock protein 70 inhibits alpha-synuclein fibril formation via interactions with diverse intermediates. J Mol Biol 2006, 364:323-336.

27. Luk KC, Mills IP, Trojanowski JQ, Lee VM: Interactions between Hsp70 and the hydrophobic core of alpha-synuclein inhibit fibril assembly. Biochemistry 2008, 47:12614-1225.

28. Neckers L, Schulte TW, Mimnaugh E: Geldanamycin as a potential anticancer agent: its molecular target and biochemical activity. Invest New Drugs 1999, 17:361-373.

29. Luo W, Dou F, Rodina A, Chip S, Kim J, Zhao Q, Moulick K, Aguirre J, Wu N, Greengard P, Chiosis G: Roles of heat shock protein 90 in maintaining and facilitating the neurodegenerative phenotype in tauopathies. Proc Natl Acad Sci USA 2007, 104:9511-9516

30. Dickey CA, Kamal A, Lundgren K, Klosak N, Bailey RM, Dunmore J, Ash P, Shoraka S, Zlatkovic J, Eckman CB, et al:: The high-affinity HSP90-CHIP complex recognizes and selectively degrades phosphorylated tau client proteins. J Clin Invest 2007, 117:648-658.

31. Lu Y, Ansar S, Michaelis ML, Blagg BS: Neuroprotective activity and evaluation of Hsp90 inhibitors in an immortalized neuronal cell line. Bioorg Med Chem 2009, 17:1709-1715.

32. Sittler A, Lurz R, Lueder G, Priller J, Lehrach H, Hayer-Hartl MK, Hartl FU, Wanker EE: Geldanamycin activates a heat shock response and inhibits huntingtin aggregation in a cell culture model of Huntington's disease. Hum Mol Genet 2001, 10:1307-1315.

33. Winklhofer KF, Reintjes A, Hoener MC, Voellmy R, Tatzelt J: Geldanamycin restores a defective heat shock response in vivo. J Biol Chem 2001 276:45160-45167.

34. McLean PJ, Klucken J, Shin Y, Hyman BT: Geldanamycin induces Hsp70 and prevents alpha-synuclein aggregation and toxicity in vitro. Biochem Biophys Res Commun 2004, 321:665-669.

35. Auluck PK, Bonini NM: Pharmacological prevention of Parkinson disease in Drosophila. Nat Med 2002, 8:1185-1186.

36. Shen HY, He JC, Wang Y, Huang QY, Chen JF: Geldanamycin induces heat shock protein 70 and protects against MPTP-induced dopaminergic neurotoxicity in mice. J Biol Chem 2005, 280:39962-39969.

37. Waza M, Adachi H, Katsuno M, Minamiyama M, Sang C, Tanaka F, Inukai A, Doyu M, Sobue G: 17-AAG, an Hsp90 inhibitor, ameliorates polyglutamine-mediated motor neuron degeneration. Nat Med 2005, 11:1088-1095

38. Fujikake N, Nagai Y, Popiel HA, Okamoto Y, Yamaguchi M, Toda T: Heat shock transcription factor 1-activating compounds suppress polyglutamine-induced neurodegeneration through induction of multiple molecular chaperones. J Biol Chem 2008, 283:26188-26197.

39. Thomas M, Harrell JM, Morishima Y, Peng HM, Pratt WB, Lieberman AP Pharmacologic and genetic inhibition of hsp90-dependent trafficking reduces aggregation and promotes degradation of the expanded glutamine androgen receptor without stress protein induction. Hum Mol Genet 2006, 15:1876-1883

40. Westerlund M, Hoffer B, Olson L: Parkinson's disease: Exit toxins, enter genetics. Progress Neurobiol 2010, 90:146-56.

41. Wang L, Xie C, Greggio E, Parisiadou L, Shim H, Sun L, Chandran J, Lin X, Lai C, Yang WJ, et al: The Chaperone Activity of Heat Shock Protein 90 is Critical for Maintaining the Stability of Leucine Rich Repeat Kinase 2. J Neurosci 2008, 28:3384-3891.

42. Moriwaki Y, Kim YJ, Ido Y, Misawa H, Kawashima K, Endo S, Takahashi R: L347P PINK1 mutant that fails to bind to Hsp90/Cdc37 chaperones is 
rapidly degraded in a proteasome-dependent manner. Neurosci Res 2008, 61:43-48.

43. Falsone SF, Kungl AJ, Rek A, Cappai R, Zangger K: The molecular chaperone Hsp90 modulates intermediate steps of amyloid assembly of the Parkinson-related protein alpha-synuclein. J Biol Chem 2009, 284:31190-31199

44. Kabuta T, Furuta A, Aoki S, Furuta K, Wada K: Aberrant interaction between Parkinson diseaseassociated mutant UCH-L1 and the lysosomal receptor for chaperone-mediated autophagy. Biol Chem 2008, 283:23731-23738

45. Kosik KS, Shimura H: Phosphorylated tau and the neurodegenerative foldopathies. Biochim Biophys Acta 2005, 1739:298-310.

46. Lau LF, Schachter JB, Seymour PA, Sanner MA: Tau protein phosphorylation as a therapeutic target in Alzheimer's disease. Curr Top Med Chem 2002, 4:395-415.

47. Goedert M, Jakes R: Mutations causing neurodegenerative tauopathies. Biochim Biophys Acta 2005, 1739:240-250.

48. Dou F, Chang X, Ma D: Hsp90 Maintains the Stability and Function of the Tau Phosphorylating Kinase GSK3 $\beta$. Int J Mol Sci 2007, 8:51-60.

49. Tortosa E, Santa-Maria I, Moreno F, Lim F, Perez M, Avila J: Binding of Hsp90 to Tau Promotes a Conformational Change and Aggregation of Tau Protein. J Alzheimers Dis 2009, 17:319-325.

50. Taldone T, Gozman A, Maharaj R, Chiosis G: Targeting Hsp90: small molecule inhibitors and their clinical development. Curr Opin Pharmacol 2008, 8:370-374.

doi: 10.1186/1750-1326-5-24

Cite this article as: Luo et $\mathrm{al}$., Heat shock protein 90 in neurodegenerative diseases Molecular Neurodegeneration 2010, 5:24

Submit your next manuscript to BioMed Centra and take full advantage of:

- Convenient online submission

- Thorough peer review

- No space constraints or color figure charges

- Immediate publication on acceptance

- Inclusion in PubMed, CAS, Scopus and Google Scholar

- Research which is freely available for redistribution

Submit your manuscript at www.biomedcentral.com/submit
Ciomed Central 